\title{
Genetic Analysis and QTL Mapping of Resistance to Gummy Stem Blight in Cucumis sativus Seedling Stage
}

Shulin Liu, Yanxia Shi, Han Miao, Min Wang, Baoju Li, Xingfang Gu, and Shengping Zhang, Institute of Vegetables and Flowers, Chinese Academy of Agricultural Sciences, Beijing, 100081, China

\begin{abstract}
Gummy stem blight (GSB), caused by Didymella bryoniae (syn. Stagonosporopsis cucurbitacearum), is an important disease of cucumber (Cucumis sativus L.) worldwide. To better understand the resistance to GSB in cucumber seedlings, a set of $160 \mathrm{~F}_{9}$ recombinant inbred lines (RILs) and a total of 405 pairs of SSR primers were employed to detect quantitative trait loci (QTLs) conferring the resistance. Genetic analysis indicated that the resistance to GSB in PI 183967 seedlings was quantitative and mainly governed by two pairs of major QTLs and multiple minor QTLs. Six QTLs, $g s b 3.1, g s b 3.2, g s b 3.3, g s b 4.1, g s b 5.1$, and $g s b 6.1$,

for resistance to GSB in cucumber seedlings were detected. The stable locus gsb5.1 on Chr.5 was repeatedly detected in three seasons. Locus gsb5.1 accounted for the highest phenotypic variation, $17.9 \%$, and was flanked by SSR15321 and SSR07711 within the genetic distance of $0.5 \mathrm{cM}$. There were 102 candidate genes predicted in the region harboring the stable QTL $g s b 5.1$, of which seven genes were related to disease resistance. These results can provide a good base for further study and molecular markers for fine-mapping the major QTL conferring GSB resistance in cucumber.
\end{abstract}

Gummy stem blight (GSB) is a destructive disease of cucurbits, including cucumber (Cucumis sativus L.), that involves three genetically distinct species of the fungus, Stagonosporopsis cucurbitacearum (syn. Didymella bryoniae), S. citrulli, and S. caricae (Stewart et al. 2015). It affects root, stems, and leaves of cucumber, often causing losses in production of 15 to $30 \%$, and up to $80 \%$ or more in severe infections.

Gummy stem blight was first described by Fautrey and Roumeguere in France as the disease caused on cucumber by Ascochyta cucumis in 1891 (Chiu and Walker 1949; Sherf and Mac Nab 1986). In 1917, gummy stem blight was reported for the first time in the U.S. affecting watermelon fruit (Sherbakoff 1917). So far, there are no reports on the inheritance of GSB in cucumber. However, resistance in watermelon, controlled by a single recessive gene $(d b / d b)$, was detected using GSBresistant PI 189225 and GSB-susceptible 'Charleston Gray' watermelons (Citrullus lanatus (Thunb.) Matsum. \& Nakai (Norton 1979). Amand and Wehner (2001) reasoned that the major effect conferred by $d b / d b$ is complemented by the expression of modifier genes, and that genetic factors are weaker than environmental factors.

In melons (Cucumis melo L.), five independent single genes conferring resistance to GSB were identified from different cultigens, four of these being dominant and one recessive (Frantz 2004; Wako et al. 2002; Zuniga et al. 1999).

As for molecular markers and genetic mapping of resistance to GSB in cucurbitaceae, Joseph (2009) identified four amplified fragment length polymorphism (AFLP) markers linked to GSB resistance in the melon cultivar PI 420145 with genetic distances of 2.0, 6.0, 5.4, and $6.0 \mathrm{cM}$. Molecular markers CMCT505, CMTC160a+b220, ISSR-57560, ISSR-100900, and CMTA170a have been closely linked with the melon GSB-resistance genes $G s b-1, G s b-2, G s b-3$, and $G s b-4$. Among these markers, CMTA170a and $G s b-4$ had the shortest genetic distance of $5.14 \mathrm{cM}$. The $S b-x$

Corresponding authors: Shengping Zhang, zhangshengping@caas.cn; XingFang Gu, guxingfang@caas.cn

Shulin Liu and Yanxia Shi contributed equally to this work.

*The $\boldsymbol{e}$-Xtra logo stands for "electronic extra" and indicates that four supplementary tables and one supplementary figure are available online.

Accepted for publication 10 March 2017

C 2017 The American Phytopathological Society
GSB-resistance gene in melon was identified and mapped to the LG4 linkage group using simple sequence repeats (SSR) markers and a double haploid (DH) melon population. A gene for resistance $(S b-1)$ was positioned to the melon LG1 linkage by Ha et al. (2010). These results provide valuable references for GSB-resistance molecular markers identification in cucumbers.

To date, there are no reports on molecular markers and genetic mapping of resistance to GSB in cucumber. In this study, a set of recombinant inbred lines (RILs) derived from the cross of wildtype and cultivated cucumbers with distinct resistance to GSB was used to conduct genetic analysis and quantitative trait locus (QTL) mapping. The aim of this study is to understand the inheritance of GSB resistance, and to map the genes controlling GSBresistance, so as to facilitate fine mapping and molecular cloning of resistance genes leading to molecular marker-assisted selection (MAS) breeding.

\section{Materials and Methods}

Plant materials. The wild-type GSB-resistant cucumber accession PI 183967 (Cucumis sativus var. hardiwickii (Royle) Alef.) was crossed with the cultivated GSB-susceptible cucumber accession designated 931 (C. sativus var. sativus). After single-seed descent (SSD) reproduction, a population was developed that consisted of $160 \mathrm{~F}_{9}$ recombinant inbred lines (RILs).

Disease-resistance screen. The seedlings were arranged randomly with three replications of six plants each. Seeds of the two parental lines, PI 183967 and 931, the $\mathrm{F}_{1}$, and 160 RILs were soaked in $5 \% \mathrm{NaOCl}$ for $10 \mathrm{~min}$, washed with tap water, and germinated in an incubator at $28^{\circ} \mathrm{C}$. Germinations were carried out in spring 2013, autumn 2013, and spring 2014. When the average radicle length was $5 \mathrm{~mm}$, the seeds were sown individually into plastic pots filled with a steam-sterilized propagation substrate composed of peat, vermiculite, and soil from a vegetable field (2:1:1, volume basis) that had been steam-sterilized at $134^{\circ} \mathrm{C}$ for $30 \mathrm{~min}$.

The pathogen for inoculation was a specific strain Ascochyta citrullina of Didymella bryoniae. This was grown on cucumber fruits for 7 to 10 days to allow pycnidia to form. Spores were scraped from the infested fruits, suspended in $10 \mathrm{ml}$ of sterile water, and the concentration adjusted to $1 \times 10^{6}$ spores $/ \mathrm{ml}$ in a hemocytometer. Seedlings were inoculated when the second true leaf was fully open by spraying all leaves uniformly on both sides using a hand pumped sprayer (YH-038, YUANHUA PENWU Factory, Taizhou, Zhejiang, China). They were then kept in dark for $24 \mathrm{~h}$ with $100 \%$ relative humidity (RH), and during the next 7 to 10 days, maintained at 80 to 
$100 \% \mathrm{RH}$ and a constant temperature of 20 to $23^{\circ} \mathrm{C}$ with a $16 \mathrm{~h}$ light photoperiod.

Resistance assessment. Ten days after inoculation, all true leaves on each plant were assessed for the degree of infection using a disease index (DI) based on Zhang et al. (2013a):

$$
\mathrm{DI}=100 \times \frac{\sum(\text { Number of plants with disease rating } \times \text { Disease rating })}{(\text { Total number of plants } \times \text { Highest disease rating possible })}
$$

The disease rating scale for each seedling was as follows: $0=$ absence of GSB spots, $1=\leq 5 \%$ GSB spots, $3=>5 \%$ but $\leq 25 \%$ GSB spots, $5=>25 \%$ but $\leq 50$ GSB spots, $7=>50 \%$ but $\leq 75 \%$ GSB spots, $9=>75 \%$ GSB spots or the whole leaf dead. A schematic diagram of the disease rating scale is shown in Figure 1, where the lines with the DI range of $0<\mathrm{DI} \leq 55$ was classified as GSBresistant, and $55<$ DI $\leq 100$ was classified as GSB-susceptible.

Genetic analysis software and statistical analysis. The DI of each plant was recorded and summarized in Microsoft Excel 2003 and SAS 9.0 software. The mean DI of each line was calculated, and the genetic parameters of the parents, $F_{1}$, and RILs were calculated. A joint analysis, assuming major genes plus the polygene model of RILs (Gai et al. 2003), was used for genetic analysis of the GSB resistance. The work was divided into four steps. The first step was to establish the genetic models. The second step was to estimate the iterated expectation and conditional maximization algorithm. The third step was to select the best genetic model through AIC (Akaike information criterion) value. The fourth step was to evaluate goodness-of-fit through the least squares method. The best genetic model was then used for estimation of other genetic parameters.

Molecular marker analysis. Young leaf tissue of the parents, the $\mathrm{F}_{1}$, and each line in the RILs population was used to extract total DNA (Maniatis et al. 1982; Staub et al. 1996). The concentration of DNA was estimated after electrophoresis on $1 \%$ agarose gels with $1 \times$ TEA buffer and staining with ethidium bromide. The PCR components and amplified conditions were as described by Zhang et al. (2013b). Three microliters of the amplified products were separated on $6 \%$ polyacrylamide gels for $1 \mathrm{~h}$ and silver stained as described by Sambrook and Russell (2001).

Polymorphisms in the parental lines, PI 183967 and 931, were detected using 2,112 pairs of SSR primers (Ren et al. 2009). Polymorphic markers were used to genotype individual lines of the RIL population for linkage map construction and QTL analysis.

Linkage construction and QTL mapping. Linkage groups (LGs) were developed with JoinMap 4.0 (Van Ooijen 2006) using a minimum logarithm of odds (LOD)-likelihood score of 2.5. The Kosambi map function (Kosambi 1944) was used to calculate the genetic distance between markers. Common markers between the present map and the high-resolution cucumber genetic map produced by Ren et al. (2009) were used to assign LGs to the seven chromosomes of cucumber. The averages of DI values for each year were used to estimate QTLs by multiple-QTL modeling (MQM) with MapQTL 4.0 (Van Ooijen et al. 2000) with composite interval mapping (CIM) analysis (Zeng 1994). Permutation tests were conducted 1,000 times to assess the LOD threshold at $\alpha=0.05$ level. QTLs above the signification threshold determined by the permutation tests were considered signification. The abbreviation of the GSB followed by the chromosomal locations and the locus number is the same as the name of each detected QTL.

Bioinformatics analysis in the genomic region harboring the stable QTL. Using the whole genome sequence of inbred line 9930 (Huang et al. 2009), sequence annotation and gene prediction were performed on the genomic regions located as the stable QTL. An E-value cutoff of $1 \times \mathrm{e}^{-10}$ to $1 \times \mathrm{e}^{-20}$ with BLASTN was used when the DNA sequences were aligned to the reference genome. Gene prediction and annotation were performed only for retained sequences with an identity of $\geq 95 \%$ as reported by Zhang et al. (2013a).

\section{Results}

GSB resistance in cucumber seedlings for the progeny of PI $183967 \times 931$. The disease indices for the GSB resistant parent PI 183967 in spring 2013, autumn 2013, and spring 2014 were $18.98 \pm 1.39(\mathrm{SE}), 29.44 \pm 2.42(\mathrm{SE})$, and $17.10 \pm 9.01(\mathrm{SE})$, respectively, with a mean of $21.84 \pm 3.84(\mathrm{SE})$; the disease index for the GSB susceptible parent in the three seasons were $67.41 \pm 2.67$ (SE), $84.17 \pm 4.63(\mathrm{SE})$, and $64.20 \pm 0.60(\mathrm{SE})$, respectively, with a mean of $71.93 \pm 6.19$ (SE) (Table 1). The disease indices for the $F_{1}$ seedlings in the three assessments were $43.95 \pm 3.49$ (SE), $61.62 \pm 3.26$ (SE), and $51.00 \pm 3.74(\mathrm{SE})$, respectively, with a mean of $52.19 \pm 5.14$ (SE) (Table 1). The frequency distributions of the disease indices for GSB in the RIL population in the three trials showed normal

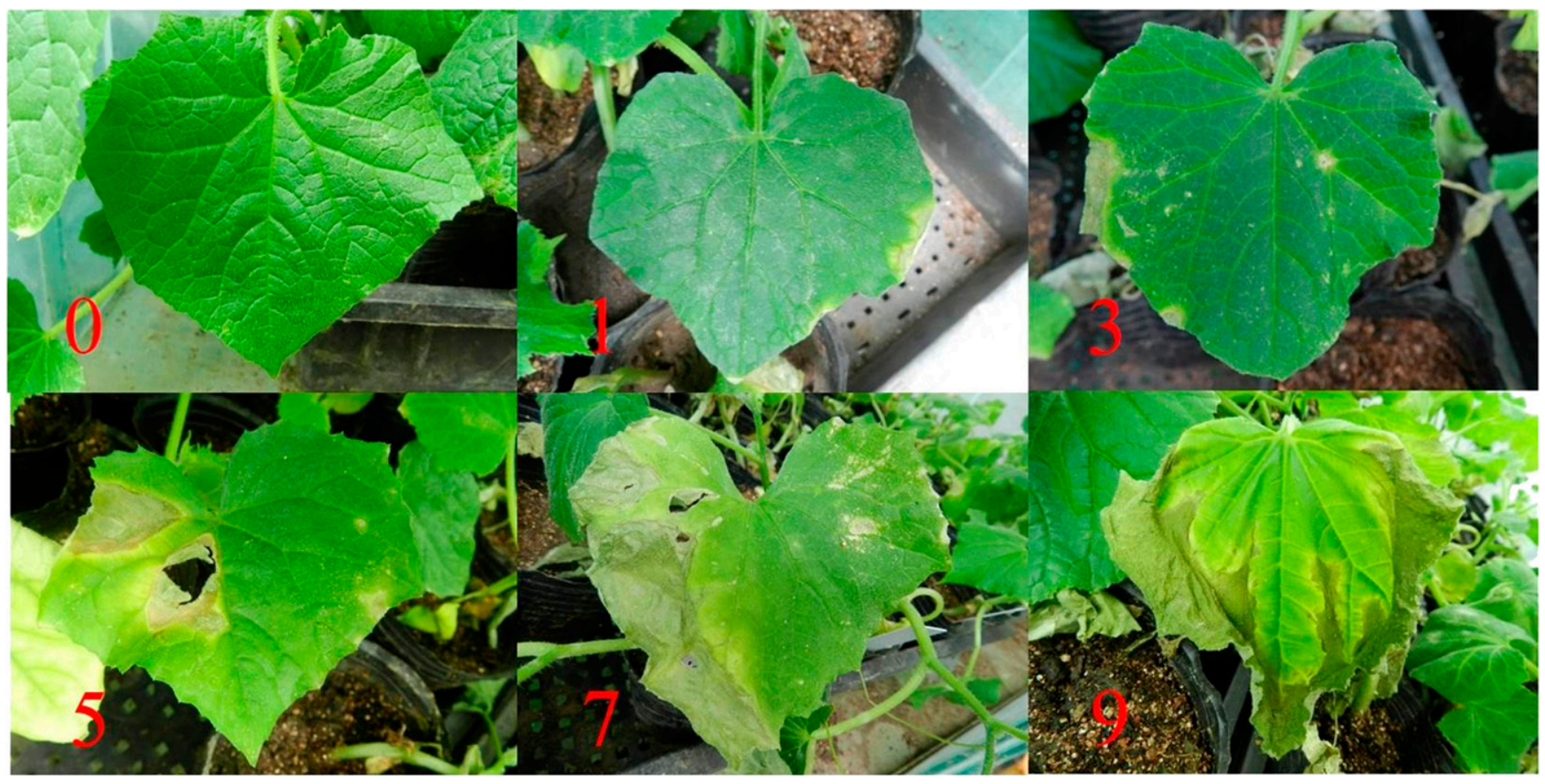

Fig. 1. Disease rating scale of each seedling was corresponding to the symptoms of all leaves. $0=$ absence of GSB spots; $1=\leq 5 \%$ GSB spots; $3=>5 \%$ but $\leq 25 \%$ GSB spots; $5=>25 \%$ but $\leq 50$ GSB spots; $7=>50 \%$ but $\leq 75 \%$ GSB spots; $9=>75 \%$ GSB spots or the whole leaf dead. 
distributions ranging from resistant to susceptible phenotypes (Fig. 2). In addition, the disease indices for PI 183967, 931, and $F_{1}$ had significant positive correlation in the three seasons, and the correlation coefficient was up to 0.983 .

Inheritance of GSB resistance in the cucumber seedlings. A joint analysis, assuming major genes plus the polygene model of RILs, together with the DI measurement in the three trials, was used to generate 38 mixed genetic models. The maximum likelihood (ML) and defined AIC of these models were estimated. Based on the principle that the smallest AIC value is the best-fitting genetic model, three models (F-3, E-2-0, and B-1-3) were prioritized based on the spring 2013 data and the lowest AIC was selected and further tested for goodness-of-fit (Tables 2 and 3). The number of statistically different parameters were 5, 3, and 8 for F-3, E-2-0, and B-1-3, respectively. Since model E-2-0 had the lowest number, it was considered the best fit for the spring 2013 GSB resistance inheritance analysis. The AIC values of E-1-9, E-2-9, and E-2-6 for the autumn 2013 data were relatively lower than other models, and therefore they were

Table 1. Gummy stem blight disease index of cucumber parental lines PI 183967 and 931, PI $183967 \times 931 \mathrm{~F}_{1}$, and some genetic factors in the RIL population for three seasons

\begin{tabular}{|c|c|c|c|c|c|c|c|}
\hline \multirow[b]{2}{*}{ Season } & \multicolumn{2}{|c|}{ Parental lines $($ mean $\pm \mathrm{SE})$} & \multirow[b]{2}{*}{ F1 } & \multicolumn{4}{|c|}{ RIL population } \\
\hline & PI183967 & 931 & & Mean & $\mathbf{S D}^{\mathbf{a}}$ & Kurtosis & Skewness \\
\hline Spring 2013 & $18.98 \pm 1.39$ & $67.41 \pm 2.67$ & $43.95 \pm 3.49$ & 55.49 & 15.38 & 0.13 & -0.60 \\
\hline Autumn 2013 & $29.44 \pm 2.42$ & $84.17 \pm 4.63$ & $61.62 \pm 3.26$ & 62.86 & 13.79 & -0.53 & 0.06 \\
\hline Spring 2014 & $17.10 \pm 9.01$ & $64.20 \pm 0.60$ & $51.00 \pm 3.74$ & 50.51 & 13.92 & -0.16 & -0.54 \\
\hline
\end{tabular}

a SD: standard deviation.
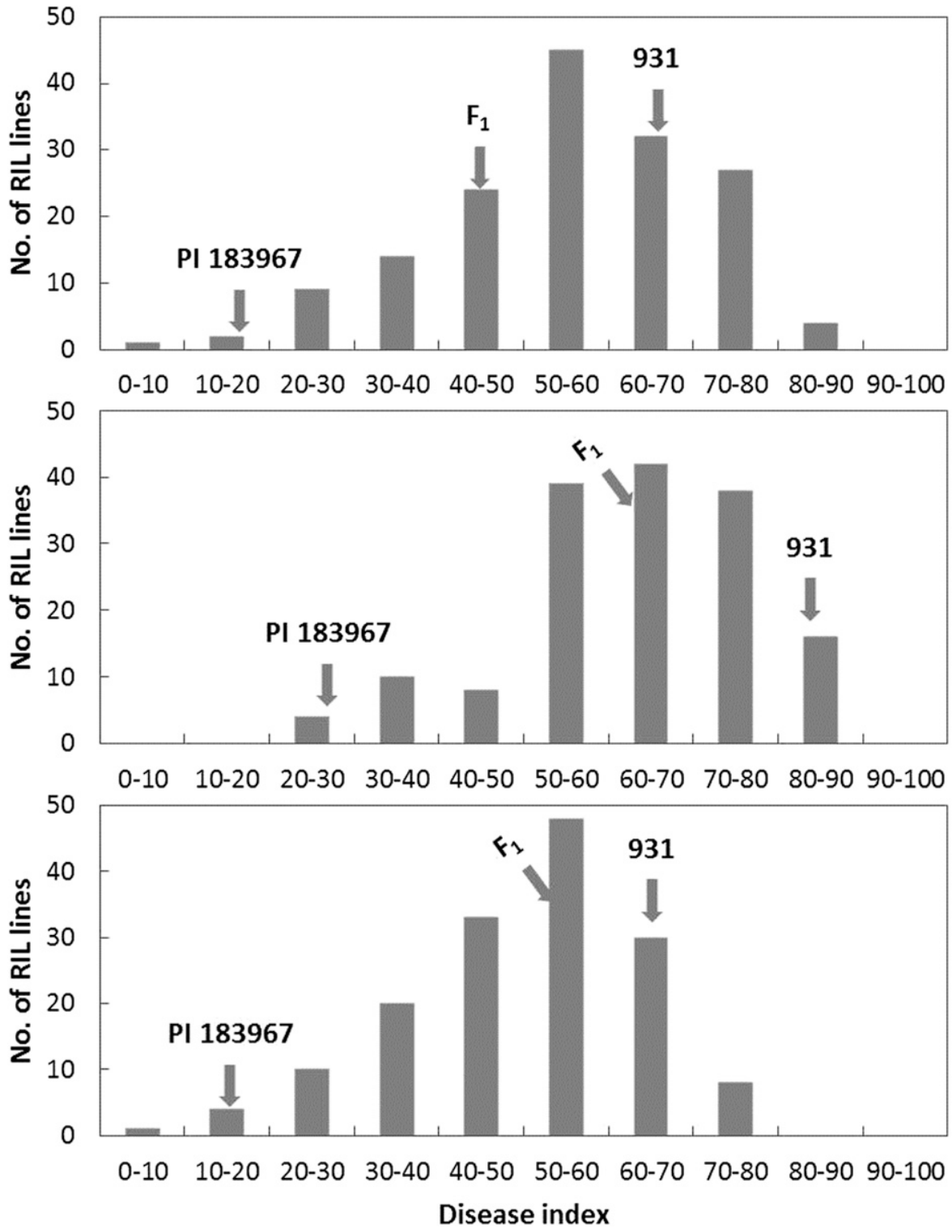

Fig. 2. Frequency distributions of gummy stem blight severity (index) of cucumber in the PI $183967 \times 931$ RIL population. The frequency distributions in spring 2013 (upper), autumn 2013 (middle), and spring 2014 (lower) trials each had a normal distribution ranging from resistant to susceptible phenotype. The mean index ratings for the parental lines and $\mathrm{F}_{1}$ are indicated by arrows. 
selected for goodness-of-fit testing (Table 3 ). The number of statistically different parameters was 2, 1, and 1 for the E-1-9, E-2-9, and E-2-6 models, respectively. Since model E-2-9 had the lowest number, it was considered the best fit model for analysis of the autumn 2013 GSB resistance inheritance. The same method was used to derive the best fit model E-2-6 for analysis of the spring 2014 GSB resistance inheritance (Tables 2 and 3).

Taken together, the best fit models for the inheritance of resistance to GSB in cucumber seedlings in spring 2013, autumn 2013, and spring 2014 were E-2-0, E-2-9, and E-2-6, respectively. The first order genetic parameters are shown in Table 4 . The test of the best fit models indicated that two pairs of major genes and multiple minor genes were controlling GSB resistance in cucumber seedlings. The heritability of the two major genes was $61.79,52.47$, and $77.27 \%$, respectively, for spring 2013, autumn 2013, and spring 2014.

QTL mapping of GSB resistance in cucumber seedlings. To identify polymorphisms between the two parental lines, 2,112 pairs of SSR primers were screened and of these, 1,125 (53.3\%) were selected for further testing. We selected 405 markers that evenly distributed on the seven cucumber chromosomes (55 per chromosome except for 75 on Chr.6) to construct the genetic map in the RIL population. Seven linkage groups were corresponded to the seven cucumber chromosomes. The total genome length coverage was $993.3 \mathrm{cM}$ with an average distance of $3.23 \mathrm{cM}$ between markers (Wang et al. 2015) (Fig. 3).

QTL mapping was conducted using this linkage map. In spring 2013, one QTL for GSB resistance in cucumber seedlings, gsb5.1, was detected. The QTL was detected on Chr.5 and was placed in the marker interval between SSR15321 and SSR07711, with an LOD of 5.56, phenotypic variation of $15.1 \%$, and additive effect of -6.91 (Table 5, Fig. 3).

In autumn 2013, three QTLs for GSB resistance were detected, which were $g s b 3.1, g s b 3.2$, and $g s b 5.1$. QTLs $g s b 3.1$ and $g s b 3.2$ were located on Chr.3 at $5.0 \mathrm{cM}$ and $43.0 \mathrm{cM}$, between SSR02451-SSR07456 and
SSR18541-SSR20863, respectively. QTLs gsb3.1 and gsb3.2 provided additive effects of 3.91 and -4.31 , and contributed to the GSB resistance phenotype by $6.0 \%(\mathrm{LOD}=2.50)$ and $7.4 \%(\mathrm{LOD}=$ 3.52), respectively. The QTL gsb5.1 was located on Chr.5 between

Table 3. Akaike information criterion values of different genetic models for the resistance to gummy stem blight in cucumber seedlings for three seasons

\begin{tabular}{lcc}
\hline Season & Model code & AIC value \\
\hline Spring 2013 & F-3 & $1,411.293$ \\
& E-2-0 & $1,412.484$ \\
Autumn 2013 & B-1-3 & $1,415.827$ \\
& E-1-9 & $1,281.233$ \\
Spring 2014 & E-2-9 & $1,282.735$ \\
& E-2-6 & $1,284.477$ \\
& F-3 & $1,285.416$ \\
& E-1-6 & $1,289.324$ \\
& E-2-6 & $1,291.244$ \\
\hline
\end{tabular}

Table 4. Parameter estimation of the first order is shown for the optimal model for resistance to gummy stem blight in cucumber seedlings for three seasons

\begin{tabular}{lccccc}
\hline Season & Model code & Content & Mean & Weight & Content ANAVA \\
\hline Spring 2013 & E-2-0 & 1 & 22.21 & 0.17 & 89.31 \\
& & 2 & 53.25 & 0.32 & \\
& & 3 & 54.36 & 0.32 & \\
Autumn 2013 & \multirow{2}{*}{ E-2-9 } & 1 & 70.67 & 0.18 & \\
Spring 2014 & & 2 & 51.01 & 0.31 & 108.72 \\
& E-2-6 & 1 & 29.72 & 0.24 & 56.69 \\
& & 2 & 62.23 & 0.52 & \\
\hline
\end{tabular}

Table 2. Goodness-of-fit of alternative genetic models for resistance to gummy stem blight in cucumber accession PI 183967 seedlings for three seasons ${ }^{\mathrm{a}}$

\begin{tabular}{|c|c|c|c|c|c|c|c|}
\hline Season & Model & Populations $^{\mathbf{b}}$ & $U 1^{2}$ & $U 2^{2}$ & $U^{2}$ & ${ }_{n} W^{2 \mathrm{c}}$ & $D_{n}^{\mathrm{d}}$ \\
\hline \multirow[t]{9}{*}{ Spring 2013} & \multirow[t]{3}{*}{ F-3 } & P1 & $0.081(0.77639)^{\mathrm{e}}$ & $0.547(0.45949) *$ & $3.455(0.06307)^{*}$ & 0.206 & 0.507 \\
\hline & & $\mathrm{P} 2$ & $2.890(0.08915)^{*}$ & $2.545(0.11067)^{*}$ & $0.041(0.83931)$ & 0.373 & 0.707 \\
\hline & & RILs & 0.017 (0.89612) & $0.116(0.73391)$ & $0.729(0.39310)^{*}$ & 0.034 & 0.042 \\
\hline & \multirow[t]{3}{*}{ E-2-0 } & P1 & $0.003(0.95625)$ & $0.140(0.70859)$ & $2.916(0.08773)^{*}$ & 0.118 & 0.388 \\
\hline & & $\mathrm{P} 2$ & $0.180(0.67121)$ & $0.669(0.41330)^{*}$ & $2.652(0.10344)^{*}$ & 0.165 & 0.51 \\
\hline & & RILs & $0.071(0.78964)$ & $0.083(0.77313)$ & $0.014(0.90455)$ & 0.022 & 0.031 \\
\hline & \multirow[t]{3}{*}{ B-1-3 } & $\mathrm{P} 1$ & $0.906(0.34129)^{*}$ & $1.576(0.20931)^{*}$ & $1.786(0.18146)^{*}$ & 0.285 & 0.627 \\
\hline & & $\mathrm{P} 2$ & $1.164(0.28063)^{*}$ & $1.564(0.21105)^{*}$ & $0.679(0.40985)^{*}$ & 0.217 & 0.592 \\
\hline & & RILs & $0.552(0.45752)^{*}$ & $0.073(0.78695)$ & $3.227(0.07245)^{*}$ & 0.235 & 0.083 \\
\hline \multirow[t]{9}{*}{ Autumn 2013} & \multirow[t]{3}{*}{ E-1-9 } & P1 & $0.037(0.84693)$ & $0.012(0.91355)$ & $0.098(0.75400)$ & 0.07 & 0.328 \\
\hline & & $\mathrm{P} 2$ & $0.216(0.64239)$ & $0.547(0.45937) *$ & $1.348(0.24561)^{*}$ & 0.113 & 0.44 \\
\hline & & RILs & $0.086(0.76932)$ & $0.016(0.89916)$ & $0.396(0.52939)$ & 0.06 & 0.058 \\
\hline & \multirow[t]{3}{*}{ E-2-9 } & $\mathrm{P} 1$ & $0.000(0.99313)$ & $0.003(0.95495)$ & $0.037(0.84724)$ & 0.076 & 0.343 \\
\hline & & $\mathrm{P} 2$ & $0.107(0.74332)$ & $0.332(0.56470)$ & $1.072(0.30055)^{*}$ & 0.099 & 0.409 \\
\hline & & RILs & $0.002(0.96413)$ & $0.006(0.93830)$ & $0.018(0.89225)$ & 0.04 & 0.057 \\
\hline & \multirow[t]{3}{*}{ E-2-6 } & $\mathrm{P} 1$ & $0.038(0.84579)$ & $0.044(0.83397)$ & $0.007(0.93211)$ & 0.081 & 0.38 \\
\hline & & $\mathrm{P} 2$ & $0.034(0.85304)$ & $0.155(0.69365)$ & $0.737(0.39077)^{*}$ & 0.085 & 0.373 \\
\hline & & RILs & $0.004(0.95213)$ & $0.006(0.93820)$ & $0.006(0.93812)$ & 0.043 & 0.059 \\
\hline \multirow[t]{9}{*}{ Spring 2014} & \multirow[t]{3}{*}{ F-3 } & $\mathrm{P} 1$ & $1.064(0.30241)^{*}$ & $0.446(0.50432)^{*}$ & $1.751(0.18572)^{*}$ & 0.163 & 0.507 \\
\hline & & $\mathrm{P} 2$ & $0.597(0.43987)^{*}$ & $1.280(0.25795)^{*}$ & $2.351(0.12516)^{*}$ & 0.263 & 0.609 \\
\hline & & RILs & $0.994(0.31874)^{*}$ & $1.320(0.25054)^{*}$ & $0.540(0.46259)^{*}$ & 0.199 & 0.081 \\
\hline & \multirow[t]{3}{*}{ B-1-1 } & $\mathrm{P} 1$ & $1.927(0.16503)^{*}$ & $1.088(0.29694)^{*}$ & $1.452(0.22821)^{*}$ & 0.225 & 0.553 \\
\hline & & $\mathrm{P} 2$ & $0.049(0.82469)$ & $0.066(0.79677)$ & $3.565(0.05901)^{*}$ & 0.218 & 0.499 \\
\hline & & RILs & $0.297(0.58567)$ & $0.160(0.68915)$ & $0.261(0.60914)$ & 0.075 & 0.047 \\
\hline & \multirow[t]{3}{*}{ E-2-6 } & $\mathrm{P} 1$ & $0.026(0.87172)$ & $0.153(0.69606)$ & $0.878(0.34865)^{*}$ & 0.052 & 0.316 \\
\hline & & $\mathrm{P} 2$ & $0.502(0.47873)^{*}$ & $0.084(0.77211)$ & $2.512(0.11298)^{*}$ & 0.247 & 0.569 \\
\hline & & RILs & $0.000(0.98525)$ & $0.001(0.97370)$ & $0.004(0.95190)$ & 0.04 & 0.048 \\
\hline
\end{tabular}

${ }^{\text {a }} U 1^{2}, U 2^{2}$, and $U 3^{2}$ are the statistic of uniformity test; ${ }_{n} W^{2}$ is the statistic of Smirnov test; $D_{n}$ is the statistic of Kolmogorov test.

${ }^{\text {b }} \mathrm{P}_{1}$ : PI 183967; $\mathrm{P}_{2}$ : 931; RILs: recombinant inbred lines.

${ }_{n} W_{0.05}^{2}=0.4610$

${ }^{\mathrm{d}} D_{n 0.05}=1.3600$.

e The relevant probability of $U 1^{2}, U 2^{2}$, and $U 3^{2}$ is shown in parentheses. * indicates that the value is significant at $P \leq 0.05$. 
SSR15321 and SSR07711, with a LOD value of 8.01 and phenotypic variation of $17.9 \%$.

Four QTLs for GSB resistance in cucumber seedling were detected in spring 2014. The QTL $g s b 3.3$ on Chr.3 was flanked by SSR12383 and SSR18541 at genetic distances of 33.0 and $40.4 \mathrm{cM}$, respectively. This QTL explained 5.7\% of the total phenotypic variance with a LOD of 3.01. On chromosome 4, the QTL ( $g s b 4.1$ ) was detected and accompanied by SSR21051 and SSR21501 with an LOD of 3.03 and an $R^{2}$ of $6.4 \%$. The QTL $g s b 5.1$ was also detected in this season with an LOD value of 4.17 and phenotypic variation of $8.1 \%$. Locus $g s b 6.1$, accounting for $6.5 \%$ of the phenotypic variance with an LOD of 3.31, was mapped to Chr. 6 at the position of $166.6 \mathrm{cM}$.

In summary, a total of six QTLs were identified for GSB resistance in the PI $183967 \times 931$ RIL population tested over three trials in this study. The QTL gsb5.1 was detected in different seasons and accounted for the highest phenotypic variation to GSB resistance (Fig. 4).

Gene prediction and annotation in the stable QTL genome region. In the cucumber $9930 \mathrm{draft}$ genome, $61 \mathrm{NBS}$ and LRR type resistance gene analogs (RGAs) were identified and were distributed mostly in 11 clusters (Huang et al. 2009). In this study, gene prediction and annotation was performed in the genomic region harboring the stable QTL ( $g s b 5.1$ ) associated with GSB resistance. Of the 102 genes predicted in this region, there were seven disease resistance-related genes and three (Csa5M467390, Csa5M467900, and Csa5M485190) belonged to the NBS-LRR gene. The three NBS-type RGAs will be candidate genes for further research associated with GSB resistance.
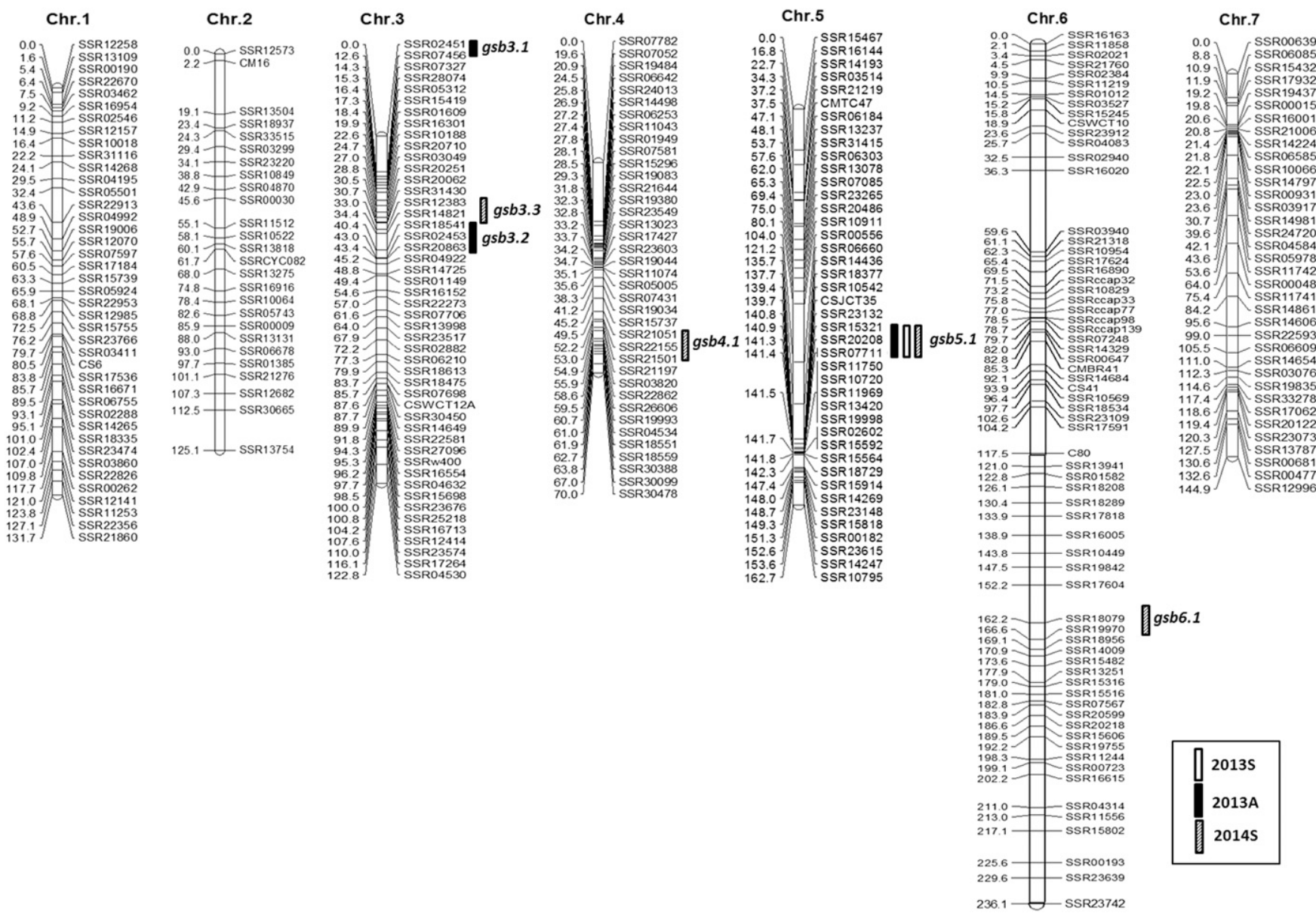

$g s b 6.1$

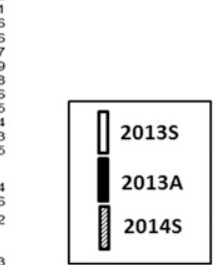

Fig. 3. Construction and chromosomal quantitative trait locus (QTL) mapping for gummy stem blight resistance in a cucumber RIL seedling population using SSR markers. Six QTLs for gummy stem blight resistance were detected in three different seasons of screening for resistance in the $\mathrm{F}_{9}$ RIL population: gsb5.1 was detected in spring 2013; gsb3.1, gsb3.2, and gsb5.1 in autumn 2013; and gsb3.3, gsb4.1, gsb5.1, and gsb6.1 in spring 2014. The loci of gsb3.1, gsb3.2, and gsb3.3 were on chromosome 3 (Chr.3) and gsb4.1, gsb5.1, and gsb6.1 were on Chr.4, Chr.5, and Chr.6, respectively.

Table 5. Quantitative trait locus (QTL) analysis of gummy stem blight resistance genes in cucumber seedlings for three seasons

\begin{tabular}{|c|c|c|c|c|c|c|c|}
\hline Season & QTL $^{\mathbf{a}}$ & Chromosome & Position(cM) & Marker interval & LOD & $\mathbf{R}^{2}(\%)$ & $\mathbf{A E}$ \\
\hline Spring 2013 & gsb5.1 & 5 & 141.3 & SSR15321-SSR07711 & 5.56 & 15.1 & -6.91 \\
\hline \multirow[t]{3}{*}{ Autumn 2013} & gsb3.1 & 3 & 5 & SSR02451-SSR07456 & 2.5 & 6 & 3.91 \\
\hline & $g s b 3.2$ & 3 & 43 & SSR18541-SSR20863 & 3.52 & 7.4 & -4.31 \\
\hline & gsb5.1 & 5 & 141.3 & SSR15321-SSR07711 & 8.01 & 17.9 & -7.35 \\
\hline \multirow[t]{4}{*}{ Spring 2014} & $g s b 3.3$ & 3 & 34.4 & SSR12383-SSR18541 & 3.01 & 5.7 & -3.55 \\
\hline & $g s b 4.1$ & 4 & 52.2 & SSR21051-SSR21501 & 3.33 & 6.4 & -3.92 \\
\hline & gsb5.1 & 5 & 141.3 & SSR15321-SSR07711 & 4.17 & 8.1 & -4.64 \\
\hline & gsb6.1 & 6 & 166.6 & SSR18079-SSR18956 & 3.31 & 6.5 & -3.72 \\
\hline
\end{tabular}

${ }^{\text {a }}$ One ( $\left.g s b 5.1\right)$, three ( $g s b 3.1, g s b 3.2$, and $\left.g s b 5.1\right)$ and four ( $g s b 3.3, g s b 4.1, g s b 5.1$, and $\left.g s b 6.1\right)$ QTLs were detected in spring 2013, autumn 2013, and spring 2014, respectively. For all seven QTLs except for $g s b 3.1$, the additive effect were negative value showing the QTL favorable allele came from the parent PI 183927. For QTL gsb3.1, the QTL favorable allele came from the parent 931. 

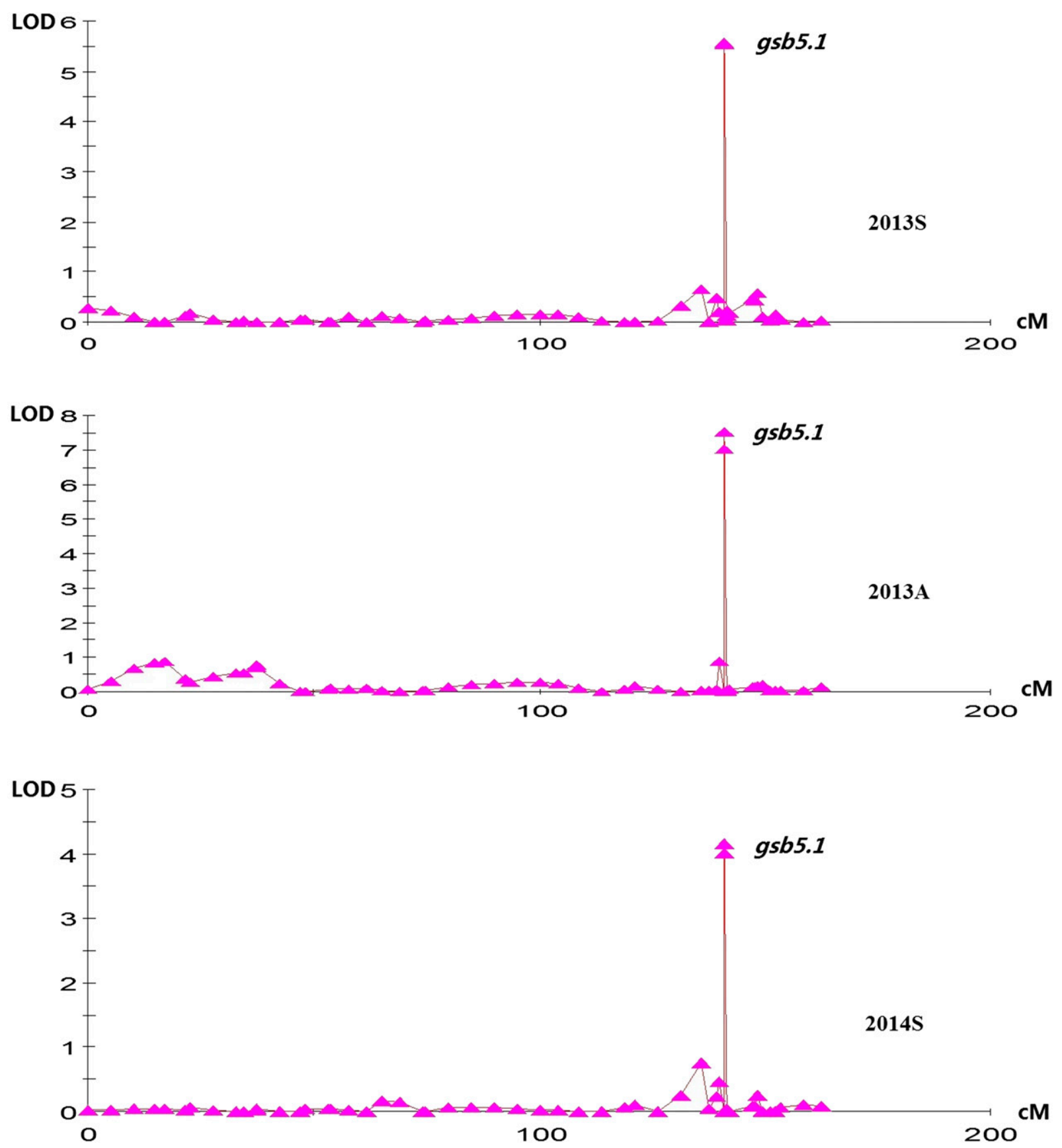

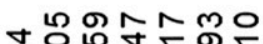

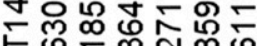

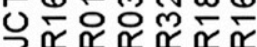

の

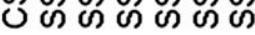

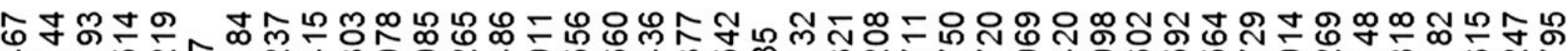

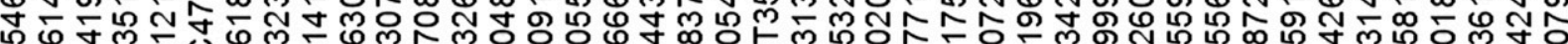

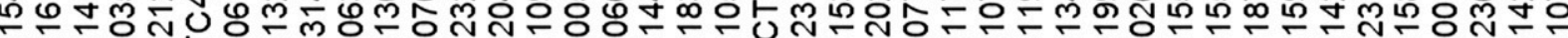

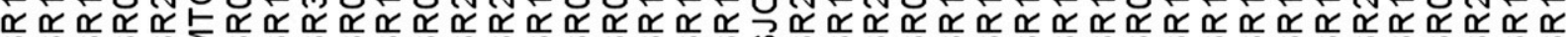

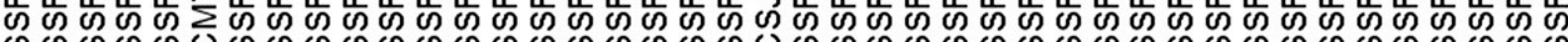

10

产

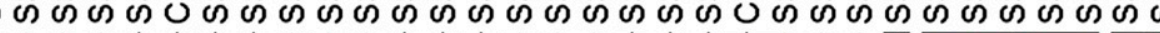

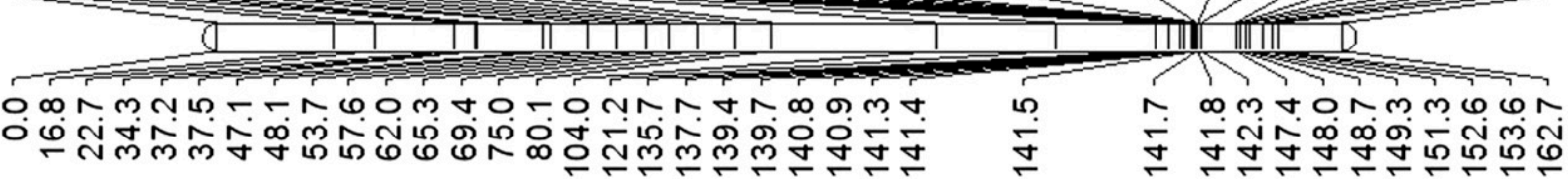

Fig. 4. The stable QTL (gsb5.1) for resistance to gummy stem blight in PI $183967 \times 931 \mathrm{~F}_{9}$ RIL population seedlings was detected on chromosome 5 . The QTL was detected in spring 2013 (upper), autumn 2013 (middle), and spring 2014 (lower) trials with a highest LOD value between SSR15321 and SSR07711. 
Table 6. Annotation and gene prediction for stable quantitative trait locus (QTL) region for resistance to gummy stem blight in seedlings of cucumber accession PI 183967

\begin{tabular}{|c|c|c|}
\hline Candidate $\mathbf{R}$ genes & Function of $\mathbf{R}$ gene & Type of $\mathbf{R}$ gene \\
\hline Csa5M467390 & IPR003590 Leucine-rich repeat ribonuclease inhibitor subtype & leucine rich repeat (LRR) \\
\hline Csa5M467900 & IPR003590 Leucine-rich repeat ribonuclease inhibitor subtype & leucine rich repeat (LRR) \\
\hline Csa5M485190 & $\begin{array}{l}\text { IPR001611 Leucine-rich repeat; } \\
\text { IPR003591 Leucine-rich repeat typical subtype; } \\
\text { IPR013210 Leucine-rich repeat-containing N-terminal }\end{array}$ & leucine rich repeat (LRR) \\
\hline Csa5M471090 & $\begin{array}{l}\text { IPR001878 Zinc finger CCHC-type; } \\
\text { IPR004859 Putative 5-3 exonuclease; } \\
\text { IPR013084 Zinc finger CCHC retroviral-type; }\end{array}$ & Zinc finger \\
\hline Csa5M477610 & $\begin{array}{l}\text { IPR007087 Zinc finger C2H2-type; } \\
\text { IPR013087 Zinc finger C2H2-type/integrase DNA-binding }\end{array}$ & Zinc finger \\
\hline Csa5M484625 & $\begin{array}{l}\text { IPR000767 Disease resistance protein; } \\
\text { IPR002182 NB-ARC }\end{array}$ & Disease resistance protein \\
\hline Csa5M494390 & $\begin{array}{l}\text { IPR000157 Toll-Interleukin receptor; } \\
\text { IPR000767 Disease resistance protein; } \\
\text { IPR001611 Leucine-rich repeat; } \\
\text { IPR002182 NB-ARC; } \\
\text { IPR003593 ATPase AAA+ type core }\end{array}$ & Toll-Interleukin receptor \\
\hline
\end{tabular}

${ }^{a}$ NB-ARC: nucleotide-binding adaptor shared by APAF-1, R proteins, and CED-4 (www.plantphysiol.org/content/140/4/1233.abstract).

\section{Discussion}

In the present study, we used an RIL population derived from PI $183967 \times 931$ and 405 pairs of SSR primers to identify candidate genes for GSB resistance in the cucumber seedlings. The frequency distributions of the disease indices suggested that the GSB resistant phenotype of parental line PI 183967 has quantitative-trait heritability characteristics rather than conferred by a single gene. So far, research progress on the genetic analysis of GSB resistance is very slow and limited in cucumber. The reasons may lie in the following four aspects. Firstly, the inheritance of GSB resistance appears to be complex in cucumber. Secondly, different cultivars or breeding lines have been used as resistance sources in different studies. Thirdly, it is difficult to compare research results because of different inoculation methods and rating scales in previous studies. Fourthly, variable environmental conditions can strongly affect the initiation and development of cucumber GSB, so it is crucial to maintain stable symptom assessment conditions during testing. In this study, we used the GSB resistant parent PI 183967 and kept the seedlings in a growth chamber at stable humidity and constant temperature after inoculation. This ensures the accuracy and repeatability of the experimental results.

Molecular marker-assisted selection (MAS) for resistance may be a more effective and efficient way compared with phenotypic selection in a conventional cucumber resistance-breeding program because a great deal of time and labor is needed and multiple tests with several replications and years are required. Therefore, MAS for GSB resistance breeding as one of the major objectives in cucumber should be enforced (Van der Meer et al. 1978; Wehner et al. 1996). In our study, we detected six QTLs for GSB resistance in cucumber seedlings. Based on its stable performance, contribution level, and LOD, gsb5.1 was postulated to be one of the stable QTLs for GSB resistance and was mapped in the region between SSR15321 and SSR07711 with genetic distance of $0.5 \mathrm{cM}$ on Chr.5. On Chr.3, three QTLs were detected associated with GSB resistance: $g s b 3.1$ was flanked by SSR02451 and SSR07456 within $12.6 \mathrm{cM}$; $g s b 3.2$ was linked to the makers SSR18541 and SSR20863 with genetic distance of $3.0 \mathrm{cM}$; $g s b 3.3$ was flanked by SSR12383 and SSR18541 within $7.4 \mathrm{cM} ; g s b 4.1$ was located in the region between SSR21051 and SSR21501 with genetic distance of $3.5 \mathrm{cM}$; $g s b 6.1$ was detected between SSR18079 and SSR18956 within $6.9 \mathrm{cM}$. These results will lay a good foundation for future studies of GBS resistance in cucumber.

In recent years, with the release of cucumber genome and melon genome, comparative genomics research saw rapid development. Previous studies had shown that cucumber Chr.3 was syntenic to melon chromosome IV and VI, cucumber Chr.5 was syntenic to melon chromosome IX and X, and cucumber Chr.6 was syntenic to melon chromosome III, VIII, and IX (Huang et al. 2009; Fukino et al. 2010; Li et al. 2011). For gummy stem blight resistance genes in melon, only $G s b-1$ was located to melon chromosome I, which was syntenic to cucumber Chr.7. In the present study, no QTL was detected on cucumber Chr.7, so we did not find QTL located in the syntenic regions of cucumber and melon.

Biological analysis was performed to predict GSB resistance related genes in the SSR15321-SSR07711 region. BLAST analysis showed that this region had a physical distance of $1,075.5 \mathrm{~kb}$ and contained 102 annotated genes based on the gene annotation. These genes were mainly classified as DNA binding site, transcriptional factors, protein kinase, cell signal transduction factor, transmembrane protein, leucine rich repeat, Zinc finger, et al. Many genes conferring resistance ( $R$ genes) to a diverse array of pathogens, including bacteria, fungi, oomycetes, viruses, and nematodes, have been isolated in plants. Nucleotide binding site (NBS) type and leucine-rich repeat (LRR) domains are encoded in the major largest $R$ gene family (Dangl and Jones 2001; Hulbert et al. 2001; Meyers et al. 2003). Among predicted genes, there are seven annotated genes related to resistance to cucumber disease, including three leucine rich repeat (LRR) resistance genes (Csa5M467390, Csa5M467900, and Csa5M485190), two Zn-finger motifs genes (Csa5M471090 and Csa5M477610), one disease resistance protein gene (Csa5M484625), and one Toll-Interleukin receptor gene (Csa5M494390) (Table 6). The large number of genes in this region makes it difficult to determine the specific cucumber gene(s) that have large effects on GSB resistance. However, these predicted GSB resistance-related genes will be key candidates for further study.

\section{Acknowledgments}

We thank Dr. Graham Collins, formerly of the University of Adelaide, South Australia, for proofreading. This study was supported by the National Natural Science Foundation of China (grant no. 31272187); the Key Laboratory of Biology and Genetic Improvement of Horticultural Crops, Ministry of Agriculture, the People's Republic of China, and the earmarked fund for Modern Agro-industry Technology Research System (CARS-25).

\section{Literature Cited}

Amand, P. C., and Wehner, T. C. 2001. Generation means analysis of leaf and stem resistance to gummy stem blight in cucumber. J. Am. Soc. Hortic. Sci. 126: 95-99.

Chiu, W. F., and Walker, J. C. 1949. Physiology and pathogenicity of cucurbit black-rot fungus. Agric. Res. 78:589-615.

Dangl, J. L., and Jones, J. D. G. 2001. Plant pathogens and integrated defence responses to infection. Nature 411:826-833.

Frantz, J. D. 2004. Five independent loci each control monogenic resistance to gummy stem blight in melon (Cucumis melo L.). Theor. Appl. Genet. 108 1033-1038. 
Fukino, N., Yoshioka, Y., Sakata, Y., and Matsumoto, S. 2010. Construction of an intervarietal genetic map of cucumber and its comparison with the melon genetic map. Pages 22-25 in: Cucurbitaceae 2010 Proc. J. A. Thies, S. Kousik, and A. Levi, eds. Am Soc Hort Sci, Alexandria, VA.

Gai, J., Zhang, Y., and Wang, J. 2003. Genetic system of quantitative traits in plants. Science Press, Beijing.

Ha, K., Zhang, H., Liu, J., Liu, P., and Wang, J. 2010. Molecular location of Didymella bryoniae resistance gene carried by $4 \mathrm{G} 21$ on Cucumis melo L. Acta. Hortic. Sin. 37:1079-1084.

Huang, S., Li, R., Zhang, Z., Li, L., Gu, X., Fan, W., Lucas, W., Wang, X., Xie, B., Ni, P., Ren, Y., Zhu, H., Li, J., Lin, K., Jin, W., Fei, Z., Li, G., Staub, J. E., Kilian, A., Van der Vossen, E. A. G., Wu, Y., Guo, J., He, J., Jia, Z., Ren, Y., Tian, G., Lu, Y., Ruan, J., Qian, W., Wang, M., Huang, Q., Li, B., Xuan, Z., Cao, J., San, A., Wu, Z., Zhang, J., Cai, Q., Bai, Y., Zhao, B., Han, Y., Li, Y., Li, X., Wang, S., Shi, Q., Liu, S., Cho, W., Kim, J., Xu, Y., HellerUszynska, K., Miao, H., Cheng, Z., Zhang, S., Wu, J., Yang, Y., Kang, H., Li, M., Liang, H., Ren, X., Shi, Z., Wen, M., Jian, M., Yang, H., Zhang, G., Yang, Z., Chen, R., Liu, S., Li, J., Ma, L., Liu, H., Zhou, Y., Zhao, J., Fang, X., Li, G., Fang, L., Li, Y., Liu, D., Zheng, H., Zhang, Y., Qin, N., Li, Z., Yang, G., Yang, S., Bolund, L., Kristiansen, K., Zheng, H., Li, S., Zhang, X., Yang, H., Wang, J., Sun, R., Zhang, B., Jiang, S., Wang, J., Du, Y., and Li, S. 2009. The genome of the cucumber, Cucumis sativus L. Nat. Genet. 41:1275-1281

Hulbert, S. H., Webb, C. A., Smith, S. M., and Sun, Q. 2001. Resistance gene complexes: evolution and utilization. Annu. Rev. Phytopathol. 39:285-312.

Joseph, N. W. 2009. Identification of amplified fragment length polymorphism markers linked to gummy stem blight resistance in melon (Cucumis melo L.). HortScience 44:32-34.

Kosambi, D. D. 1944. The estimation of map distance from recombination values. Ann. Eugen. 12:172-175.

Li, D., Cuevas, H. E., Yang, L., Li, Y., Garcia-Mas, J., Zalapa, J., Jack, E. S., Luan, F., Umesh, R., He, X., Gong, Z., and Weng, Y. 2011. Syntenic relationships between cucumber (Cucumis sativus L.) and melon (C. melo L.) chromosomes as revealed by comparative genetic mapping. BMC Genomics 12:396.

Maniatis, T., Fritsch, E. F., and Sambrook, J. 1982. Molecular Cloning: A Laboratory Manual. Cold Spring Harbor Laboratory Press, Cold Spring Harbor, NY.

Meyers, B. C., Kozik, A., Griego, A., Kuang, H., and Michelmore, R. W. 2003. Genome-wide analysis of NBS-LRR-encoding genes in Arabidopsis. Plant Cell Online 15:809.

Norton, J. 1979. Inheritance of resistance to gummy stem blight [caused by Didymella bryoniae] in watermelon. HortScience 14:630-632.

Ren, Y., Zhang, Z., Liu, J., Staub, J. E., Han, Y., Cheng, Z., Li, X., Lu, J., Miao, H., Kang, H., Xie, B., Gu, X., Wang, X., Du, Y., Jin, W., and Huang, S. 2009. An integrated genetic and cytogenetic map of the cucumber genome. PLoS One 4: e5795.

Sambrook, J., and Russell, D. W. 2001. Molecular Cloning: A Laboratory Manual, 3rd Ed. Cold Spring Harbor Laboratory Press, Cold Spring Harbor, NY.

Sherbakoff, C. D. 1917. Some important diseases of truck crops in Florida. Fla. Agric. Exp. Stn. Bull. 139:269-273.

Sherf, A. F., and Mac Nab, A. A. 1986. Vegetable diseases and their control, 2nd Ed. Wiley, New York.

Staub, J. E., Bacher, J., and Poetter, K. 1996. Sources of potential errors in the application of random amplified polymorphic DNAs in cucumber. HortScience 31:262-266.

Stewart, J. E., Turner, A. N., and Brewer, M. T. 2015. Evolutionary history and variation in host range of three Stagonosporopsis species causing gummy stem blight of cucurbits. Fungal Biol. 119:370-382.

Van der Meer, Q. P., Van Bennekom, J. L., and Van der Giessen, A. C. 1978. Gummy stem blight resistance of cucumber (Cucumis sativus L.). Euphytica 27:861-864.

Van Ooijen, J. W. 2006. JoinMap ${ }^{\circledR}$ 4, software for the calculation of genetic linkage maps in experimental populations. Kyazma B.V., Wageningen, The Netherlands.

Van Ooijen, J. W., Boer, M. P., Jansen, R. C., and Maliepaard, C. 2000. MapQTL Version 4.0, Software for the Calculation of QTL Positions on Genetic Maps. Plant Research International, Wageningen, The Netherlands.

Wako, T., Sakata, Y., Sugiyama, M., Ohara, T., Ishiuchi, D., and Kojima, A. 2002. Identification of melon accessions resistant to gummy stem blight and genetic analysis of the resistance using an efficient techniquence for seeding test. Acta. Hortic. 588:161-164.

Wang, M., Liu, S., Zhang, S., Miao, H., Tian, G., Lu, H., Liu, P., Wang, Y., and $\mathrm{Gu}, \mathrm{X}$. 2015. QTL mapping of seedling traits in cucumber using recombinant inbred lines. Plant Breed. 135:124-129.

Wehner, T. C., Amand, P. C., and Lower, R. L. 1996. 'M17'gummy stem blight resistant picking cucumber inbred. HortScience 31:1248-1249.

Zeng, Z. 1994. Precision mapping of quantitative trait loci. Genetics 136 1457-1468.

Zhang, S., Liu, M., Miao, H., Zhang, S., Yang, Y., Xie, B., Wehner, T. C., and Gu, X. 2013a. Chromosomal mapping and QTL analysis of resistance to downy mildew in Cucumis sativus. Plant Dis. 97:245-251.

Zhang, S., Miao, H., Sun, R., Wang, X., Huang, S., Wehner, T. C., and Gu, X. 2013 b. Localization of a new gene for bitterness in cucumber. J. Hered. 104 134-139.

Zuniga, T. L., Jantz, J. P., Zitter, T. A., and Jahn, M. K. 1999. Monogenic dominant resistance to gummy stem blight in two melon (Cucumis melo) accessions. Plant Dis. 83:1105-1107. 